Review

\title{
Biocompatibility of Advanced Manufactured Titanium Implants-A Review
}

\author{
Alfred T. Sidambe \\ Bioengineering \& Health Technologies Group, School of Clinical Dentistry, University of Sheffield, \\ 19 Claremont Crescent, Sheffield S10 2TA, UK; E-Mail: a.t.sidambe@ gmail.com; \\ Tel.: +44-114-271-7890; Fax: +44-114-279-7050 \\ External Editor: Jung Kwon Oh
}

Received: 25 August 2014; in revised form: 5 October 2014 / Accepted: 8 December 2014 /

Published: 19 December 2014

\begin{abstract}
Titanium (Ti) and its alloys may be processed via advanced powder manufacturing routes such as additive layer manufacturing (or 3D printing) or metal injection moulding. This field is receiving increased attention from various manufacturing sectors including the medical devices sector. It is possible that advanced manufacturing techniques could replace the machining or casting of metal alloys in the manufacture of devices because of associated advantages that include design flexibility, reduced processing costs, reduced waste, and the opportunity to more easily manufacture complex or custom-shaped implants. The emerging advanced manufacturing approaches of metal injection moulding and additive layer manufacturing are receiving particular attention from the implant fabrication industry because they could overcome some of the difficulties associated with traditional implant fabrication techniques such as titanium casting. Using advanced manufacturing, it is also possible to produce more complex porous structures with improved mechanical performance, potentially matching the modulus of elasticity of local bone. While the economic and engineering potential of advanced manufacturing for the manufacture of musculo-skeletal implants is therefore clear, the impact on the biocompatibility of the materials has been less investigated. In this review, the capabilities of advanced powder manufacturing routes in producing components that are suitable for biomedical implant applications are assessed with emphasis placed on surface finishes and porous structures. Given that biocompatibility and host bone response are critical determinants of clinical performance, published studies of in vitro and in vivo research have been considered carefully. The review concludes with a future outlook on advanced Ti production for biomedical implants using powder metallurgy.
\end{abstract}


Keywords: CP-Ti; Ti6Al4V; titanium; biocompatibility; powder metallurgy; metal injection moulding; additive manufacturing; 3-D printing; cytotoxicity; implants

\section{Introduction}

Materials used for biomedical applications exist in different forms and they must possess specific properties to fulfil this role [1]. Materials such as metals are commonly used as implants and such metals have to possess properties which will enable them to function inside the human or animal body [2]. Biomaterials, as they are also known, are expected to have biomechanical properties which are comparable to those of autogenous tissues without side effects. The properties which determine whether a material is suitable for biomedical implant applications include biocompatibility, bioadhesion, biofunctionality and corrosion resistance [2]. In order to ensure safety and to have the desired results, implants and other devices intended for biomedical use are regulated by different bodies globally such as the U.S. Food and Drug Administration (FDA) and the International Standards Organisation (ISO) [3]. The main metallic biomaterials are stainless steels, cobalt alloys, titanium and titanium alloys [1]. This review has been limited to the study of titanium and its alloys because this is a metal whose widespread use has been limited by its high cost due to the multi-step Kroll extraction process of the Ti raw material [4]. Advanced powder manufacturing routes such as metal injection moulding (MIM) have emerged as techniques that can minimise the cost of titanium implant production.

\section{Titanium as Implant Material}

Titanium and titanium alloys exhibit a high specific strength [5], which makes titanium an excellent choice for biomedical applications [6]. Furthermore, titanium is considered to be biocompatible because it has a low electrical conductivity which contributes to the electrochemical oxidation of titanium leading to the formation of a thin passive oxide layer [7]. The oxide layer in turn leads to a high resistance to corrosion. This protective passive layer is retained at $\mathrm{pH}$ values of the human body [8] due to titanium having an oxide isoelectric point of 5-6 [1]. In aqueous environments $\mathrm{Ti}$ and its oxides have low ion-formation tendency and low reactivity with macromolecules [9]. Titanium alloys are used in biomedical implant devices which replace damaged hard tissue. Some examples of Ti uses in biomedical applications are dental and orthopaedic implants, artificial hearts, pacemakers, artificial knee joints, bone plates, cardiac valve prostheses, screws for fracture fixation, artificial hip joints [1] and cornea backplates [10]. Titanium and titanium alloys have therefore been used widely as biomedical implant materials since the early 1970s and the implants have been available as machined and cast components. The alloys that are preferred for the fabrication of titanium implants are commercially pure titanium (CP-Ti) and titanium alloy Ti6Al4V (Ti-64). CP-Ti has a higher resistance to corrosion and is widely regarded as the most biocompatible metal because of a stable and an inert oxide layer which spontaneously forms when its surface is exposed to oxidising media [1]. Almost all commercially available permucosal dental implants are made from CP-Ti as a result of the pioneering research of Brånemark and his co-workers [11]. 
The CP-Ti and Ti-64 manufactured via the traditional routes (such as strips, sheets, plates, bars, billets, forgings and wires) are specified according to the American Society for Testing and Materials (ASTM) as grades 1 to 5. Grades 1 to 4 are the unalloyed CP-Ti and grade 5 is the alloyed Ti-64. Table 1 summaries the mechanical properties of titanium according to the ASTM standards F67 [12] and F136 [13] for bars, billets and forgings. Grade 2 titanium is the main unalloyed Ti used in dental implant applications. Grade $2 \mathrm{Ti}$ has a minimum yield strength of $275 \mathrm{MPa}$ and this is the equivalent of yield strength in heat-treated austenitic stainless steels. Grade 5 Ti-64 is the most widely used titanium alloy in biomedical implants where high strength is required [1]. As it can be seen form Table 1, CP-Ti has lower strength whilst Ti-64 is an $\alpha+\beta$ alloy which offers a higher strength [14].

Table 1. Selected mechanical requirements properties of titanium bar for implant [1].

\begin{tabular}{cccccc}
\hline Material & Specification & $\begin{array}{c}\text { Tensile } \\
\text { strength (MPa) }\end{array}$ & $\begin{array}{c}\text { 0.2\% Proof } \\
\text { stress (MPa) }\end{array}$ & $\begin{array}{c}\text { Elongation } \\
(\%)\end{array}$ & $\begin{array}{c}\text { Elastic } \\
\text { modulus (GPa) }\end{array}$ \\
\hline CP-Ti & ASTM F67 Grade 1 & 240 & 170 & 24 & $103-107$ \\
- & ASTM F67 Grade 2 & 345 & 275 & 20 & $103-107$ \\
- & ASTM F67 Grade 3 & 450 & 380 & 18 & $103-107$ \\
- & ASTM F67 Grade 4 & 550 & 483 & 15 & $103-107$ \\
Ti6A14V & ASTM F136 Grade 5 & 860 & 795 & 10 & $114-120$ \\
\hline
\end{tabular}

$\mathrm{MPa}=$ megapascal, $\mathrm{GPa}=$ gigapascal.

As much as CP-Ti and Ti-64 have a number of advantages which have already been mentioned above, they do however have some disadvantages as far as implant applications are concerned in that they both have low wear resistance properties. There have also been concerns that the vanadium contained in the Ti-64 alloy is cytotoxic [2] which means that this alloy is limited to certain appropriate applications and devices [13]. CP-Ti and Ti-64 have an elastic modulus which is considered to be high when compared to the bone [2]. These factors therefore limit the application of CP-Ti and Ti-64 because of the mismatch of the elastic (or Young's) modulus between the implant and the bones [15]. A low elastic modulus is desirable in implants because it helps to avoid stress shielding and the associated bone resorption [16]. As a result, new titanium alloy compositions which are specifically tailored for biomedical applications have been developed. The first generation of these biomedical implant alloys have included Ti-6Al-7Nb and Ti-5Al-2.5Fe, two alloys with properties similar to Ti-64 that were developed to address concerns relating vanadium's cytotoxicity [2]. Titanium alloys containing $\mathrm{Nb}$, $\mathrm{Ta}$ and $\mathrm{Zr}$ ( $\beta$ stabilising elements) with a low modulus, high strength, resistance to corrosion and biocompatibility have also been developed for biomedical implant applications.

Another solution to the problems associated with the high titanium elastic modulus has been to use advanced manufacturing processes such as additive layer manufacturing (ALM) to make highly porous titanium structures of $\mathrm{CP}-\mathrm{Ti}$ and $\mathrm{Ti}-64$. These porous structures can be tailored to have excellent mechanical properties similar to those of human bone and are usually designed to facilitate bone ingrowth [17]. The elastic modulus of titanium is about $114 \mathrm{GPa}$ while that of cancellous and cortical bone range from $0.5 \mathrm{GPa}$ to a maximum of $20 \mathrm{GPa}$ [18]. The mechanical properties and behaviour of titanium implants can be varied via the volume fraction and size distribution of the pore structures [19] and it has been shown that the elastic modulus of porous titanium decreases with increasing pore size under a compressive force [18]. 
The increased interest in the advanced manufacturing of titanium has led to the subsequent need for regulations and standards and in response the ASTM and the International Organization for Standardization (ISO) have recently published new standards for ALM and MIM titanium. Table 2 shows the chemical composition requirements for MIM and ALM titanium according to ASTM standards F2989, F2885 [20], F2924 [21], F3001 [22] and ISO 22068 [23] standards. Titanium ASTM F2989 "MIM 1" has the highest purity with $0.20 \mathrm{wt} \%$ maximum iron content, which is lower than "MIM 2" and "MIM 3" ( $0.30 \mathrm{wt} \%$ maximum). In comparison to the chemical properties of ASTM F67, the chemical requirements are unchanged except for the oxygen in Grade MIM 3 which is set at $0.3 \mathrm{wt} \%$ maximum whereas it is $0.35 \mathrm{wt} \%$ in ASTM F67. When comparing the alloyed Ti-64 ASTM standards, the maximum oxygen level is set at $0.13 \mathrm{wt} \%$ extra-low interstitial (ELI) for the bars, billets and forgings (ASTM F136). In MIM the maximum oxygen level is $0.2 \mathrm{wt} \%$ because in processing MIM there is always oxygen pickup during sintering.

Table 2. Chemical composition requirements for metal injection moulding (MIM) and additive layer manufacturing (ALM) titanium according to American Society for Testing and Materials (ASTM) standards.

\begin{tabular}{ccccccccc}
\hline \multirow{2}{*}{ Specification } & \multicolumn{10}{c}{ Chemical composition (wt \%) } \\
\cline { 2 - 9 } & $\mathbf{A l}$ & $\mathbf{V}$ & $\mathbf{F e}$ & $\mathbf{C}$ & $\mathbf{N}$ & $\mathbf{O}$ & $\mathbf{H}$ & $\mathbf{Y}$ \\
\hline ASTM F2989 MIM 1 & - & - & $<0.2$ & $<0.08$ & $<0.03$ & $<0.18$ & $<0.015$ & - \\
ASTM F2989 MIM 2 & - & - & $<0.3$ & $<0.08$ & $<0.03$ & $<0.25$ & $<0.015$ & - \\
ASTM F2989 MIM 3 & - & - & $<0.3$ & $<0.08$ & $<0.05$ & $<0.30$ & $<0.015$ & - \\
ISO 22068 MIM Ti-400 & - & - & - & $<0.2$ & $<0.1$ & $<0.4$ & - & - \\
ASTM F2885 Grade 5 & $5.5-6.75$ & $3.5-4.5$ & $<0.30$ & $<0.08$ & $<0.05$ & $<0.20$ & $<0.015$ & $<0.005$ \\
ISO 22068 MIM-Ti6A14V-600 & $5.0-7.0$ & $3.0-5.0$ & - & $<0.2$ & $<0.1$ & $<0.4$ & - & - \\
ASTM F2924 ALM Ti6A14V & $5.5-6.5$ & $3.5-4.5$ & $<0.3$ & $<0.08$ & $<0.05$ & $<0.20$ & $<0.015$ & - \\
ASTM F3001 ALM Ti6A14V (ELI) & $5.5-6.5$ & $3.5-4.5$ & $<0.25$ & $<0.08$ & $<0.05$ & $<0.13$ & $<0.012$ & $<0.005$ \\
\hline
\end{tabular}

As it can be seen from Table 2 there are currently two alloyed Ti grades made by ALM that are in the standards and that can be used for surgical implant applications. The ASTM F2924-14 [21] and F3001-14 [22] standards cover additively manufactured Ti6A14V components using full-melt powder bed fusion such as electron beam melting and laser melting [22]. Table 3 shows the minimum mechanical properties of Ti manufactured by ALM and MIM according to ASTM F2924-14, ASTM F3001-14, ASTM F2989-11, F2885-11 and ISO 22068.

In comparison to requirements for unalloyed titanium bars, billets and forgings, the tensile strength and yield strength requirements are higher in ASTM F2989 MIM whereas the elongation requirements are lower. This is because in the as-sintered state Ti MIM carries some residual porosity [24]. The tensile strength and yield strength requirements in Ti-64 MIM are lower than in bars, billets and forgings whereas it is the same in Ti-64 ALM. The requirements for elongation are lower with Ti-64 ALM. 
Table 3. Mechanical properties of CP-Ti MIM, Ti-64 MIM and ALM.

\begin{tabular}{cccccc}
\hline Material & Specification & $\begin{array}{c}\text { Tensile strength } \\
(\mathbf{M P a})\end{array}$ & $\begin{array}{c}\mathbf{0 . 2 \%} \text { Proof } \\
\text { stress (MPa) }\end{array}$ & $\begin{array}{c}\text { Elongation } \\
(\boldsymbol{\%})\end{array}$ & $\begin{array}{c}\text { R of A * } \\
(\boldsymbol{\%})\end{array}$ \\
\hline MIM CP-Ti & ASTM F2989 MIM 1 & 370 & 315 & 23 & 25 \\
- & ASTM F2989 MIM 2 & 420 & 360 & 17 & 20 \\
- & ASTM F2989 MIM 3 & 495 & 390 & 10 & 15 \\
- & ISO 22068 MIM Ti-400 & 500 & 400 & 5 & \\
MIM Ti6A14V & ASTM F2885 Grade 5 & 780 & 680 & 10 & 15 \\
- & ISO 22068 MIM-Ti6A14V-600 & 800 & 600 & 3 & - \\
ALM Ti6A14V & ASTM F2924 ALM Ti6A14V & 895 & 825 & 10 & 15 \\
ALM Ti6Al4V & ASTM F3001 ALM Ti6Al4V (ELI) & 860 & 795 & 8 & 25 \\
\hline
\end{tabular}

$* \mathrm{R}$ of $\mathrm{A}=$ Reduction of Area.

\section{Advanced Powder Processing of Titanium}

As it was mentioned earlier, titanium production is hampered by the high cost in traditional manufacturing processes, and poor workability for complex shape production. This has led to numerous investigations of various potentially lower-cost processes [4]. Although there are various powder metallurgy routes of processing titanium as have been listed by Froes [25], this review is confined to the relatively new areas of ALM and titanium MIM.

Advanced manufacturing routes such as ALM and Ti MIM are processes which offer design flexibility and cost-saving respectively, for fabricating products that have complicated shapes with a very high accuracy of size. ALM and MIM are distinct from traditional machining techniques, which mostly rely on the removal of material by methods such as cutting or drilling (subtractive manufacturing) [26]. Thus, ALM and MIM are increasingly being employed as processes for fabricating surgical implants including orthopaedic and dental implants. With increasing demand for medical implantation, there is increasing need for implants that offer reliability, adequate mechanical properties and that offer unique properties (such as patient specific implants) as well as comfort. MIM is a processing route that offers reduction in costs, with the added advantage of near net-shape fabrication as reported by Ferri et al. [27] and Ebel et al. [28]. On the other hand ALM is used to make patient specific, complex, cellular and functional mesh arrays implants or bone substitutes [29].

The aim of this review is to summarize existing literature and report on the use of advanced manufacturing to fabricate titanium alloy implants. Emphasis is placed on the surface finishes, porous structures and various in vitro and in vivo biocompatibility studies that have been carried out on the advanced manufactured implants. The development of new processes and materials does not typically include biocompatibility testing until the prototype stage is reached. Regulation agencies such as the Food and Drug Administration (FDA) in the USA require biocompatibility testing per ISO 10993 or ASTM F748 prior to device approval [30]. Therefore there is a need to carry out biocompatibility testing in any material or new material processing method [30].

\subsection{Additive Layer Manufacturing}

Additive layer manufacturing encompasses a group of technologies that have emerged in the last decade. In ALM, the material is added one cross-sectional layer at a time to create an object [31]. 
ALM uses the additive method to make a three-dimensional solid object of almost any shape from a computer-aided design (CAD) model. The CAD file model which constitutes the part geometry is created and once optimised, the CAD file is then "sliced numerically" into the layer thickness the machine will build in. After that it is transferred to the ALM machine's software and loaded to the ALM machine allowing a file based build to begin. Layers of material are laid down successively with each layer corresponding to a different shape. Figure 1 illustrates the flow diagram of ALM system from CAD file through to component manufacture.

Figure 1. Flow diagram of ALM system from computer-aided design (CAD) file through to component manufacture.

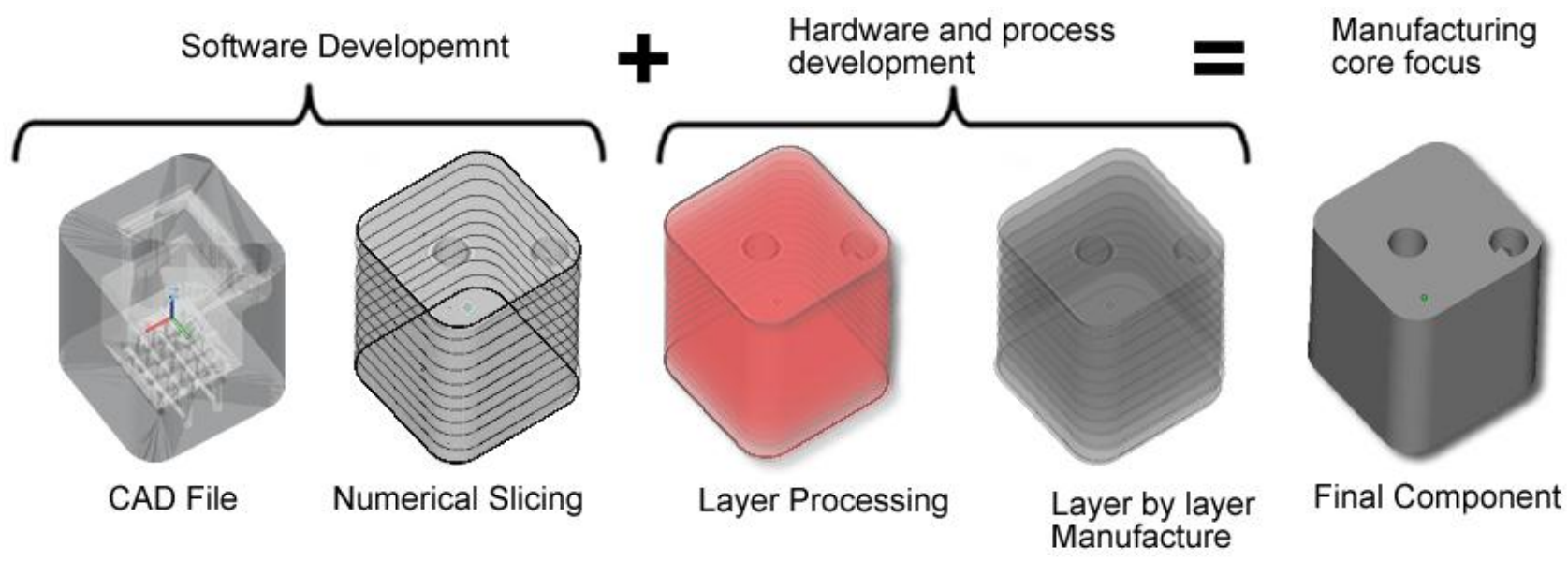

ALM technologies include fused deposition modelling, laser micro-sintering, direct metal laser sintering (DMLS), three-dimensional (3-D) laser cladding, electron beam melting (EBM), and electron beam sintering (EBS) [26]. This review looks in detail at the two systems which have the capability to manufacture Ti, namely the EBM and DMLS and briefly at other ALM techniques.

\subsubsection{Electron Beam Melting (EBM)}

Electron beam melting $(\mathrm{EBM})$ is one of the additive manufacturing techniques mainly used for metallic biomaterials. The EBM system manufactures parts by melting the metal powder layer by layer using a magnetically directed electron beam (up to $3 \mathrm{~kW}$ ) under a high vacuum atmosphere [32]. It is for this reason that EBM is particularly suited for the manufacturing of titanium and titanium alloy implants. Figure 2 is a schematic drawing of an electron beam melting system.

Part of the challenges of using EBM in the manufacturing of surgical implants is to optimise the surface finish of the final components. This highly depends on the EBM processing parameters such as beam current, part orientation and powder particle size. It is widely known and accepted that the surface topography of biomedical implants affects the biocompatibility because it influences the cell attachment, proliferation, and differentiation. The performance of biomedical implants and their biocompatibility depends very much on the initial interaction between surfaces of the implants and the biological environment [33]. As a result, there are a number of studies that have been carried out to determine the biocompatibility of titanium implants manufactured via EBM. 
Figure 2. Schematic drawing of an electron beam melting system.

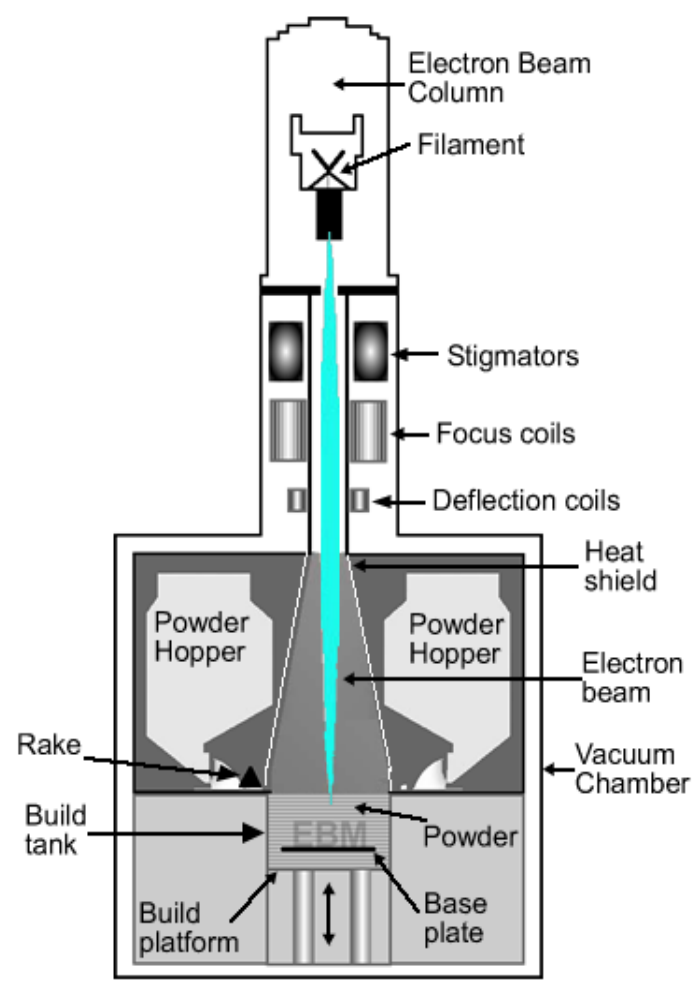

Ponader et al. [33] manufactured Ti-64 components with different surfaces and porosity using EBM and then they assessed, in vitro, the surface matrices for cell attachment, proliferation, and differentiation using human fetal osteoblasts (hFOB 1.19). In that study the cell proliferation was found to be more pronounced in the Ti-64 samples which were compact than on the porous samples. These Ti-64 compacts consisted of adherent partly molten titanium particles on the surface, leading to a surface roughness (Ra) of $\leq 24.9 \mu \mathrm{m}$. In porous samples with highly rough surfaces $(\mathrm{Ra} \geq 56.9 \mu \mathrm{m})$ there was reduced proliferation of the hFOB cells. The authors found that cell differentiation markers were not influenced by the surface roughness. Surface characteristics of titanium could easily be changed by EBM in order to further improve proliferation [33]. This study also concluded that the wear resistance must be optimized and that further investigations were necessary to clarify whether the observed in vitro biocompatibility can also be observed in vivo, where bone tissue can grow into the EBM structures. The authors then carried out a follow on study where they assessed EBM smooth compacts and porous Ti-64 structures as scaffolds for bone formation, in vivo [17]. The implants were placed into defects in the frontal skull of 15 domestic pigs. After surgery, X-Ray photographs were taken to analyse the microscopic structure of the direct contact between bone and implant surfaces in intervals of 14, 30, and 60 days. The results showed that bone ingrowth was increasing with the intervals as follows: around $14 \%$ after 14 days, $30 \%$ after 30 days and $46 \%$ after 60 days in both smooth compact and rough porous implants. There was less bone-implant contact around compact specimens $(6 \%)$ than around porous specimens (9\%) after the 60 days. In the study the authors succeeded in demonstrating the suitability of highly porous titanium implants as scaffolds for bone ingrowth especially in orthopaedic and maxillofacial applications [17].

Whilst Ponader et al. [17] in vivo study showed relatively low osseointegration within the first 60 days, another in vivo study which examined the bone-to-implant contact of EBM Ti-64 implants was 
carried out by Thomsen et al. [34] and this showed the bone-to-implant contact as $29 \%-41 \%$ after 6 weeks in rabbits. After producing EBM Ti-64 implants with an increased surface roughness, the authors machined a selected number of samples and also included conventionally produced bulk Ti-64 material in their study. The results of the early bone response in rabbits showed that the as-EBM Ti-64 implants showed no significant difference in tissue response when compared to the conventional wrought titanium alloy implants and the machined EBM Ti-64. The as-EBM Ti-64 implant specimens had an area surface roughness $\left(S_{a}\right)$ greater than $15 \mu \mathrm{m}$. Also revealed in this study were the results of the chemical and mechanical properties of the electron beam-melted Ti-64 material which were within the ASTM F136 specifications. This study was done prior to the issuing of the ASTM 3001 standard.

The biocompatibility of polished, unpolished and porous EBM Ti-64 was also studied by Haslauer et al. [29] using in vitro cell culture. The authors compared the cellular response in discs which were seeded with 20,000 human adipose-derived adult stem cells (hASCs). The assessments showed that the hASCs were alive on all discs after 8 days, that cellular proliferation on porous EBM Ti-64 discs was increased compared to solid polished and unpolished EBM discs and that the release of the pro-inflammatory cytokines (IL-6 and IL-8) was lower for porous EBM discs than for other discs. Additionally, IL-6 and IL-8 releases at day 7 were lower for porous EBM discs than for other discs. The authors were satisfied that the biocompatibility of the EBM Ti-64 discs was comparable to that of the commercial implant types.

In a study which involved the surface modification of implants via coating, Li et al. [35] fabricated porous Ti-64 implants via the EBM process after which they used the biomimetic approach to coat the surfaces. The in vitro and in vivo biocompatibilities were evaluated for implants with and without biomimetic apatite coating. The results of the in vitro biocompatibility of the EBM porous titanium were positive for cell attachment, proliferation and cell morphology. The comparison of implants with and without biomimetic coating showed that cell proliferation in porous EBM Ti-64 implants could match that of coated implants. Similarly, the in vivo histological analysis showed a comparable rate of both ingrowth and bone formation between EBM Ti-64 and coated Ti-64 after 12 weeks. The analysis of the mechanical properties showed that the EBM porous Ti-64 had a Young's modulus that is similar to cortical bone (14.5 to $38.5 \mathrm{GPa}$ ).

In a study that included another long-term in vivo experiment, Palmquist et al. [36] evaluated the osseointegration of porous EBM Ti-64 and solid EBM machined Ti-64 cylindrical and disk-shaped implants. The Ti-64 was implanted in sheep, bilaterally in the femur and subcutaneously in the dorsum. After retrieval 26 weeks later, the results showed that both porous and solid implants were osseointegrated and that high bone-implant contact of 57\% was observed throughout the porous implant. However, in discussing the effects of surface topography, the authors suggested that it is not always certain that specific biological effects can be assigned to specific surface property.

The above assertion by Palmquist et al. [36] is confirmed by the fact that, although they are interlinked, results of the biocompatibility EBM of titanium alloys listed above are varied. Cell proliferation has been shown to be more pronounced in specimens which are compact and with a lower surface roughness, whereas in another case the authors claim the EBM Ti-64 had the biocompatibility properties which matched reference samples [35] and in another case the authors found more proliferation in rougher specimens compared to the reference samples [29]. Nevertheless, the literature survey has revealed to a certain extent that EBM of titanium can be adopted for the fabrication of custom 
orthopaedic implants. The process can be used in designing implants with specific surface roughnesses and porosity. The biocompatibility studies have shown how the design flexibility can be correlated with bone ingrowth and cell proliferation, for example. The results open up the possibilities of wider use of EBM titanium to reconstruct specific bone defects.

\subsubsection{Direct Metal Laser Sintering (DMLS), Selective Laser Melting/Sintering}

Direct metal laser sintering (DMLS) is an additive metal fabrication technology that was developed by EOS who are based in Munich, Germany. DMLS is often also referred to using the terms selective laser sintering (SLS) or selective laser melting (SLM).

In the DMLS system, a high-powered optic laser with a power of $200 \mathrm{~W}$ to $400 \mathrm{~W}$ is used to fuse metal powder into a solid component based on a 3D CAD file. In a similar manner to the EBM system, components are built from layer to the next layer using the additive method with the layer thickness typically being $20 \mu \mathrm{m}$. Figure 3 shows the schematic overview of SLM form of DMLS cycle.

Figure 3. Schematic overview of selective laser melting (SLM) cycle.

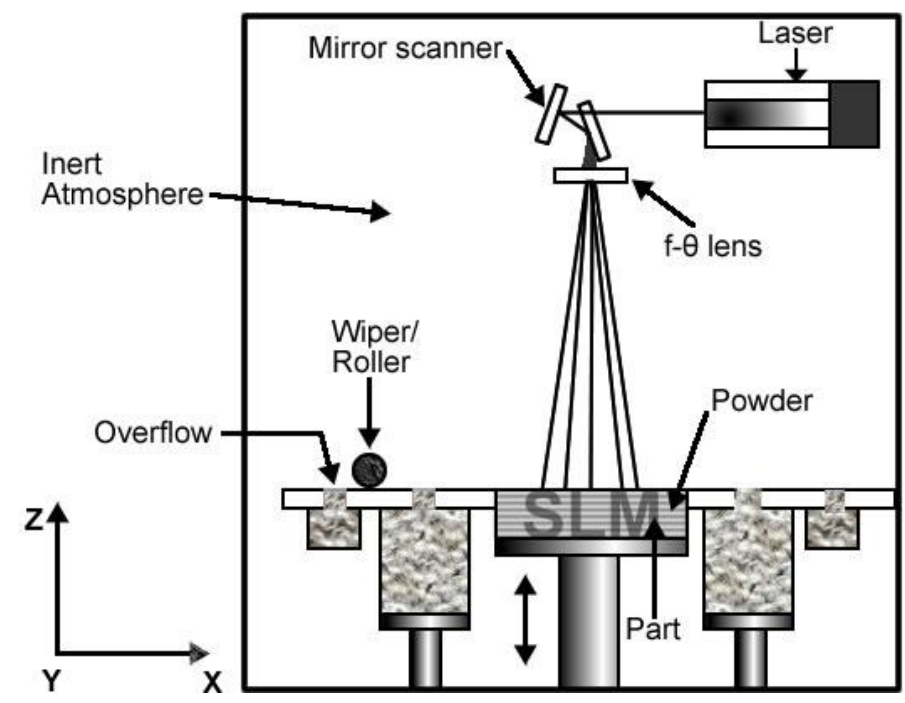

The DMLS therefore carries the challenges of manufacturing of biomedical implants with the desired surface finish without the need to post process by polishing etc. as on the EBM system. One of the studies that have been carried out in order to investigate the suitability of the titanium DMLS for biomedical implants fabrication was presented by Hollander et al. [37]. They presented results which demonstrated the effect of different surface properties on Ti-64 "bone substitutes" made via SLM. In particular, the biocompatibility of SLM Ti-64 material was studied using the primary human osteoblasts cells (HOB). Comparisons were made of the SLM surfaces with the commercial Thermanox ${ }^{\circledR}$ (Nalge Nunc Int., New York, NY, USA) control and conventional bulk titanium. The results showed that the cultured cells attached and proliferated on SLM substrates and the activity of alkaline phosphatase (AP) was also shown to increase after 7 days, but decrease after 14 days [37]. The authors concluded that the increased metabolic activity of osteoblasts on SLM discs compared to the controls may have been due to the greater surface area on the SLM material, which took longer to be covered by the cells. Figure 4 shows the example of an exact computerised tomography (CT) based complex human vertebra processed by the authors using SLM. 
Figure 4. Example of an exact CT-based part of a complex human vertebra processed by SLM using additive manufacturing [37].

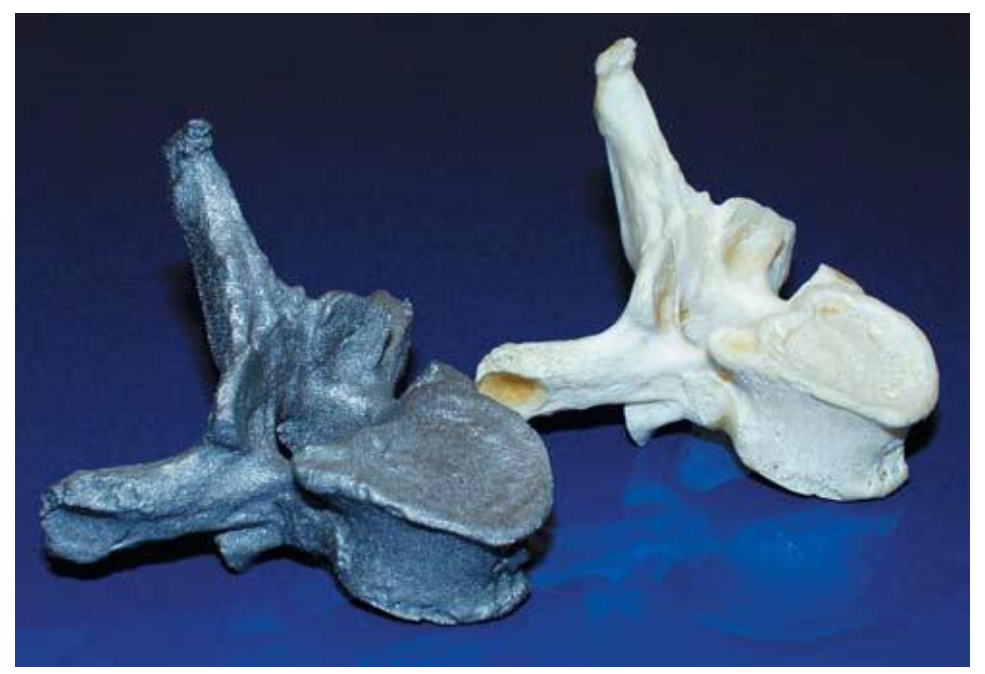

Using a DMLS variant called the Selective Laser Sintering (SLS) method, Hollister et al. [38] manufactured craniofacial and temporomandibular joint (TMJ) scaffolds for craniofacial reconstruction. The authors developed two interconnected porous architecture designs, one being interconnected spherical pores and the other being interconnected cylindrical pores whose porosity was ranging from $50 \%$ to $70 \%$. They used various biomaterials which included an unspecified grade of titanium and presented in the results initial in vitro and in vivo test data showing substantial bone ingrowth (between $40 \%$ and $50 \%$ at 6 weeks, between $70 \%$ and $80 \%$ at 18 weeks) for all scaffolds. The results of the in vivo tests showed that the scaffolds, including titanium, were able to support bone regeneration via delivery of BMP-7 transduced human gingival fibroblasts in a mouse model. Furthermore, the titanium scaffolds had mechanical property values lying in between those of cortical and trabecular bone and also supported bone and cartilage regeneration needed to reconstruct craniofacial structures.

Therefore in implantology involving metallic biomaterials like $\mathrm{Ti}$ it is important to understand the role of specific surface properties when bone is in contact with an implant. It is for this reason that studies have also been carried out to understand early human bone response to Ti implants, with the more recent studies looking at the advanced manufacturing routes such as DMLS. The early human bone response to a DMLS Ti-64 implant surface has been reported by Mangano et al. [39] after they carried out a study where micro-implant was inserted into the anterior mandible of a patient during conventional implant surgery of the jaw as part of the in vivo study. After two months of unloaded healing in this study, the micro implant and surrounding tissues were extracted and the structure of tissue was studied by microscopy. The histology showed the peri-implant bone in close contact with the surface of the implant. The mean of bone-to-implant contact was $69.51 \%$. The authors concluded that DMLS Ti-64 is a promising alternative to conventional implant surface topographies [39].

Whilst using in vitro studies to demonstrate osseointegration of DMLS titanium, Warnke et al. [40] cultured human osteoblasts on SLM-produced Ti-64 mesh scaffolds. Evaluation of the cell occlusion of pores with different widths $(0.45$ to $1.2 \mathrm{~mm})$ was carried out. The biocompatibility results showed that the osteoblasts had a well-spread morphology and also had multiple contact points. It was found that pore overgrowth increased after 6 weeks of culture where the pore widths were 0.45 and $0.5 \mathrm{~mm}$, and in 
the course of 3 weeks where the pore widths were $0.55,0.6$, and $0.7 \mathrm{~mm}$. No pore closure was observed on pores of width $0.9-1.2 \mathrm{~mm}$. The authors also found that porosity and maximum compressive load at failure increased and decreased with increasing pore width, respectively. In summary, their scaffolds were biocompatible, and pore width influenced pore overgrowth and resistance to compressive force [40].

The literature survey has revealed to a certain extent that DMLS of titanium can be adopted for the fabrication of custom orthopaedic implants. The results of in vivo tests showed that the DMLS scaffolds with unpolished and unmodified surfaces were able to support bone regeneration and that DMLS of titanium as an advanced manufacturing technology is a promising alternative to conventional implant surface topographies. On the other hand the survey has revealed that porous Ti DMLS scaffolds are biocompatible, and that pore width can influence growth around the pores and the resistance to compressive force.

\subsection{Metal Injection Moulding}

Metal injection moulding is a processing route that offers reduction in costs for Ti biomaterials and implants, with the added advantage of near net-shape components. The small to medium sized components with complex geometries that can be manufactured via MIM have the potential to be fully exploited by the implant industry.

The MIM cycle starts with preparation of a feedstock which consists of mixing fine metallic powder and a binder material. The resulting feedstock is then granulated and an injection moulding machine is used to inject the feedstock into a mould cavity under an elevated temperature $\left(<200{ }^{\circ} \mathrm{C}\right)$ and pressure. Once in the mould the molten feedstock cools and solidifies to produce a green part. The binder is then removed and what is left behind is a highly porous brown part. The brown part is then sintered and shrinks, typically to $>95 \%$ of the pore-free density (PFD) [24]. More details about MIM have been published elsewhere [41] and the flow diagram for the MIM process is shown in Figure 5.

Figure 5. Metal injection moulding flow diagram.

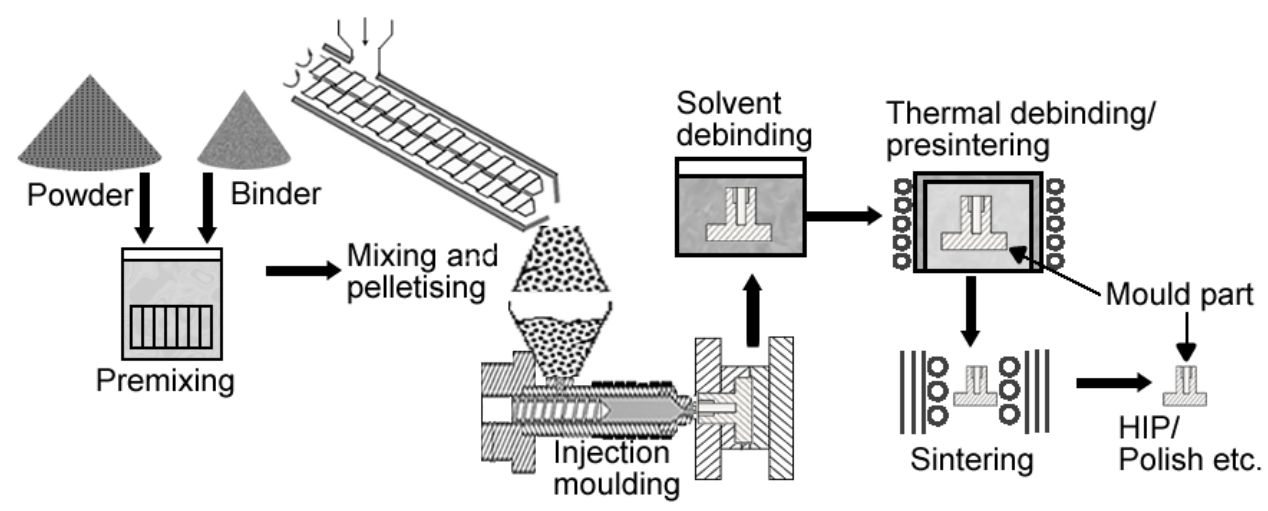

MIM in manufacturing is well established but the processing of titanium and titanium alloys in this field is still in its infancy because the process has had to depend on high quality starting powders, carbon free binders and improved furnaces for sintering in order to reduce contamination of the titanium. With the necessary improvements, the interest in the manufacturing of titanium for biomedical applications has gathered pace in recent years. Among the challenges in titanium MIM is the production of components with desirable surface finishes, mechanical and chemical properties. In MIM, the quality of 
the surface finish highly depends on the processing parameters such as mould tool finish and starting powder particle size distribution.

Among the studies that have shown the biocompatibility of implantable MIM Ti is a publication by Sago et al. [42]. In the study the authors manufactured Ti-64 devices via MIM and presented the results of the mouldability, the microstructure of the alloy, the mechanical properties as well as the biocompatibility. Their results showed that MIM can produce Ti-64 alloys which meet the specification requirements of implantable ASTM F1472 in the hot isostatically pressed (HIPped) condition. The samples were shown to have passing results for a series of biocompatibility tests, comparable to the wrought grade. Biocompatibility testing for MIM Ti-64 was carried out for hemocompatibility (ISO 10993-4), cytotoxity (ISO 10993-5), sensitization (ISO-10993-10), irritation (ISO 10993-10), systemic toxicity (ISO 10992-11), and implantation (ISO 10993-6) [42].

In a later study, Sago et al. [43] also found that the chemical, metallurgical, and mechanical properties of the MIM Ti-64 met the recently published property specifications ASTM F2885 for MIM implantable grades. The samples they manufactured were submitted to a commercial laboratory for biocompatibility testing where they passed a series of biocompatibility tests and were shown to perform at an equivalent level and with no significant differences in biocompatibility when compared with the same grade of implantable wrought alloy.

As more parts are being made from titanium alloys via MIM, the researchers are increasingly producing parts with excellent tensile properties. However, the presence of information containing the fatigue properties which could be useful for biomedical implant applications is scarce. Thus in a study that was intended to manufacture MIM titanium with improved fatigue properties, Ebel et al. [28] studied the mechanical, biological, and corrosion properties of specimens manufactured from Ti-6Al-4V-0.5B alloy. There were concerns that there may be unknown reactions in the body environment due to the boron content. Hence corrosion and biocompatibility tests were performed. The human osteosarcoma cell line MG-63 cells were cultured to perform adhesion, proliferation, and viability experiments and the results showed that the alloy $\mathrm{Ti}-6 \mathrm{Al}-4 \mathrm{~V}-0.5 \mathrm{~B}$ satisfies the requirements of a permanent implant material manufactured by MIM [28].

More studies on the suitability of MIM titanium for biomedical implant applications was carried out by Auzene et al. [44]. Their study presented results of the surface quality of MIM CP-Ti and MIM Ti-64 and its effect on biocompatibility. Comparisons were carried out between machined grade $4 \mathrm{CP}-\mathrm{Ti}$, MIM grade $4 \mathrm{CP}-\mathrm{Ti}$ and MIM grade $4 \mathrm{CP}-\mathrm{Ti}$ with three different commercial medical implant coatings (BIOCOAT ${ }^{\circledR}$, BIODIZE $^{\circledR}$ and BIOCER ${ }^{\circledR}$ : Steiger Galvanotechnique SA., Chatel-Saint-Denis, Switzerland). Thermanox ${ }^{\circledR}$ was used a control sample. The results revealed that in comparison to machined CP-Ti, MIM CP-Ti had a specific surface roughness which exhibited an excellent biological response. Cell adhesion of the cultured bone explants was poor on the Thermanox ${ }^{\circledR}$ control, and much improved on the MIM-Ti, BIODIZE ${ }^{\circledR}$ and BIOCOAT $^{\circledR}$ but did dramatically increase on BIOCER ${ }^{\circledR}$. The chemical and mechanical properties conformed to ASTM F67 standards for MIM CP-Ti and to ASTM standards F136 and F2885 for MIM Ti-64 [44].

More results of the study mentioned above were then published by Demangel et al. [45]. Their report published results of the biocompatibility of MIM CP-Ti with various anodic oxidation post-treatments as reported by Auzene et al. [44]. It was shown that MIM-Ti compared to machined CP-Ti demonstrated a specific surface topography with a higher roughness. MIM-Ti and BIOCER ${ }^{\circledR}$ samples significantly enhanced 
cell proliferation, cell adhesion and cell differentiation of bone explants compared to CP-Ti. In addition the authors performed some anodisation post-treatment in this study which demonstrated the ability to improve osseointegration through anionic modification treatment. Figure 6 shows the topographic 3D view of machined CP-Ti (a) and MIM CP-Ti (b) surfaces, $1 \times 1 \mathrm{~mm}$ studied by Demangel et al. [45].

Figure 6. Topographic 3D view of machined CP-Ti (a) and MIM CP-Ti (b) surfaces, $1 \times 1 \mathrm{~mm}[45]$.
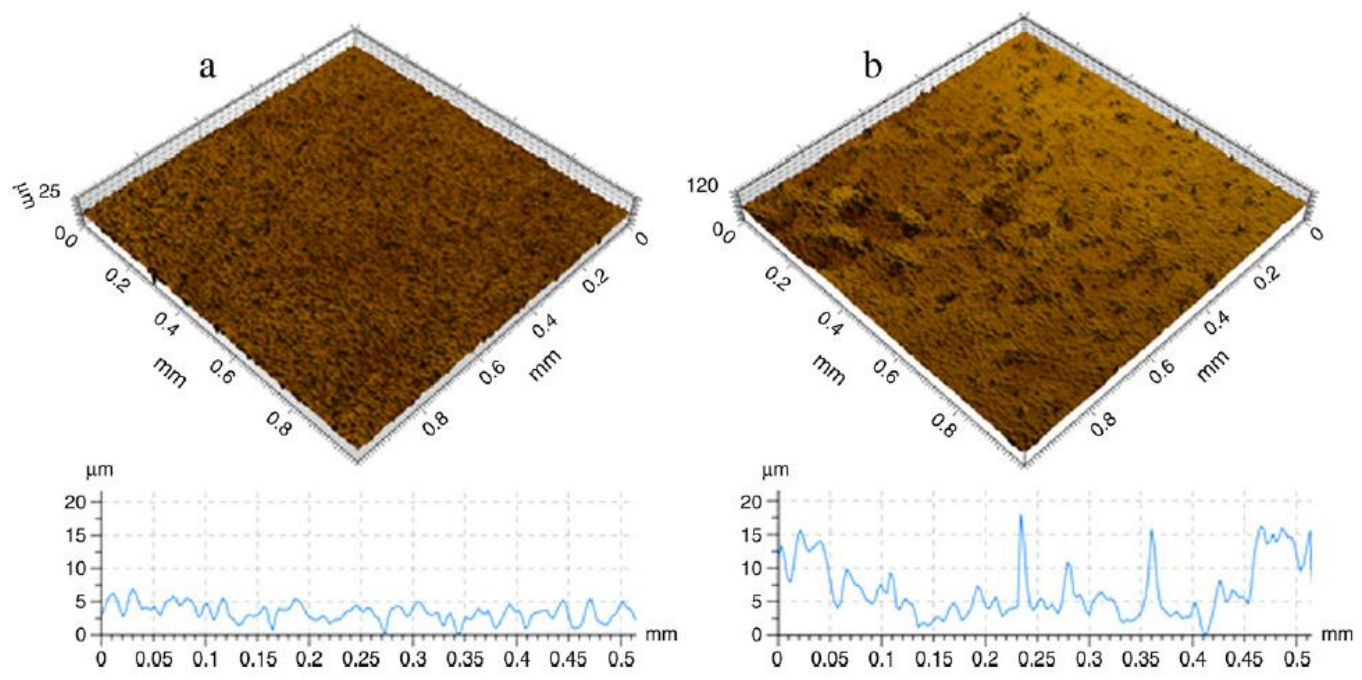

Yet more studies relating to the study of the biocompatibility of MIM Ti-64 were carried out by Ibrahim et al. [46]. Firstly, the in vitro cytotoxicity of MIM Ti-64 was carried out using the mouse fibroblast L929 cell lines. The MIM Ti-64 part was found to be non-toxic to mouse fibroblast cell lines L929. Most of the cells proliferated with numerous filopodia and were attached to the MIM Ti-64 part [46]. Secondly, Ibrahim et al. [46] carried out an in vivo test of the MIM Ti-64 by placing the implant into the mandible of Macaca fascicularis. Results showed continuous contact between surrounding tissue and the Ti-64 implant as shown in Figure 7 [46].

Figure 7. Micrograph showing surrounding tissue on the MIM Ti-64 implant showing continuous contact [46].
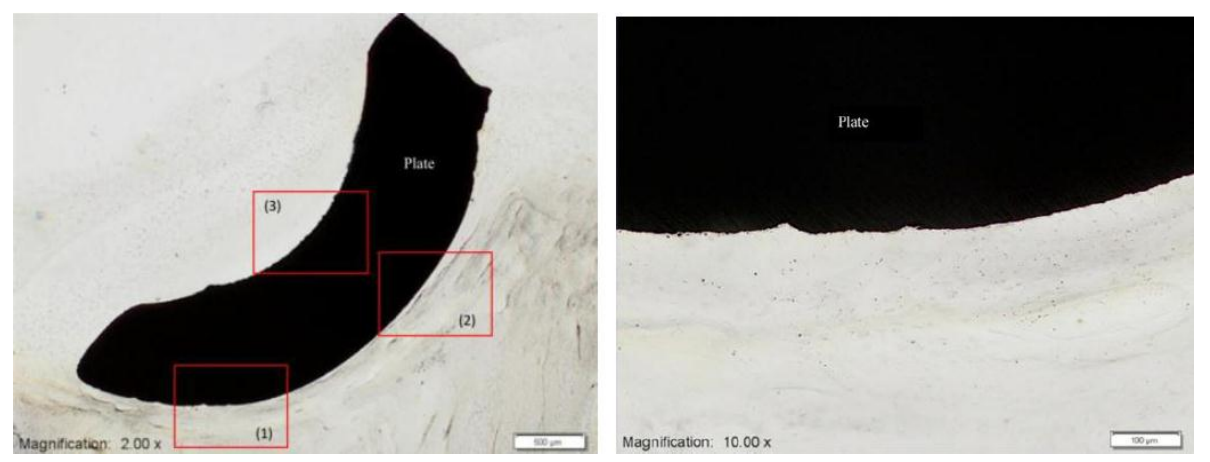

\subsection{Other Advanced Manufacturing Techniques}

There are a number of variations of additive manufacturing that have been employed by researchers to make implants. One such technique is Laser engineered net shaping or LENS $^{\mathrm{TM}}$ which is a technology 
developed by Sandia National Laboratories. Similarly to EBM and DSLM, LENS ${ }^{\mathrm{TM}}$ is used for fabricating metal parts directly from a computer-aided design (CAD) solid model. The difference is that in LENS $^{\mathrm{TM}}$ the metal powder is injected into a molten pool which is created by a focused, high-powered laser beam. Figure 8 is a schematic representation of LENS ${ }^{\mathrm{TM}}$ process.

Figure 8. Schematic representation of LENS process.

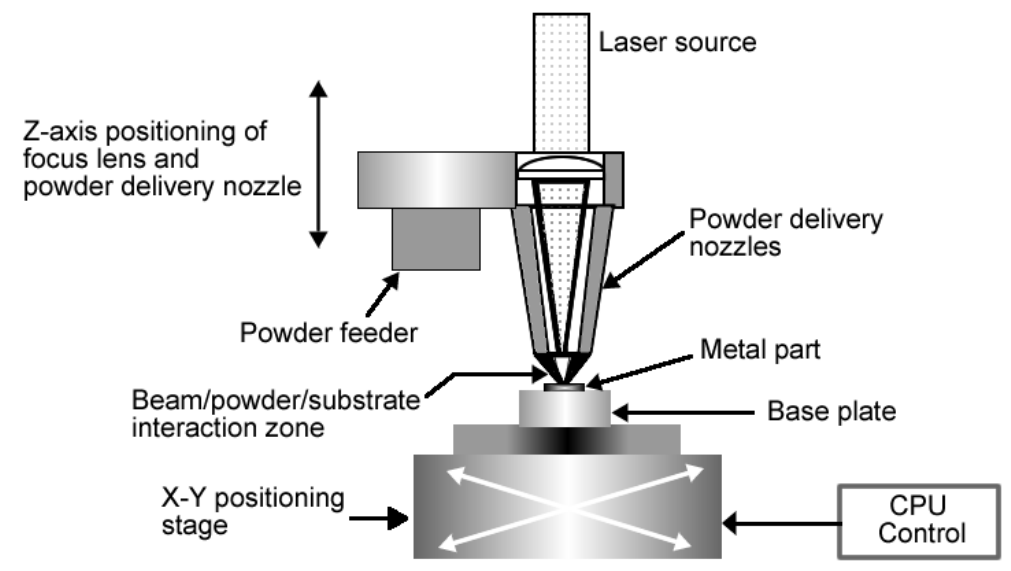

LENS $^{\mathrm{TM}}$ produced Ti parts have a rough surface with a macroporous structure and it is this property which allowed for Xue et al. [47] to use this method to fabricate porous CP-Ti implants. The effects of the porous structure on bone cell responses were evaluated in vitro using human osteoblast cells (OPC1). In the results the cells were found to be well spread on the surface of porous Ti and they formed strong local adhesion with assays showing that the porous CP-Ti surface favours bone cell proliferation. The porous CP-Ti LENS ${ }^{\mathrm{TM}}$ implants were also shown to stimulate faster OPC1 cell differentiation compared to a polished CP-Ti sheet. It was stated that this is due to the change in cell morphology within the pores of CP-Ti implant samples. The results also showed that a critical pore size of $>200 \mu \mathrm{m}$ is crucial for cell ingrowth into the pores. When the pore size is $<150 \mu \mathrm{m}$, cells were found to span directly across the pores [47].

This influence of porosity on LENS $^{\mathrm{TM}}$ manufactured titanium was also studied by Bandyopadhyay et al. [48]. After fabricating porous Ti-64 structures, using the LENS ${ }^{\mathrm{TM}}$ technology, the authors sought to demonstrate that advanced manufacturing techniques such as LENS ${ }^{\mathrm{TM}}$ can be used to fabricate low-modulus implants, with tailored porosity and which are capable of achieving long-term in vivo stability. At a porosity of 23-32 vol \%, the corresponding effective elastic modulus was tailored between 7 and $60 \mathrm{GPa}$, equivalent to human cortical bone. The in vivo tests carried out on male Sprague-Dawley rats for 16 weeks revealed that there was significant increase in calcium within the implants, which is an indicator of excellent biological tissue ingrowth through interconnected porosity. Results also showed that the total amount of porosity influences the role in tissue ingrowth [47]. Figure 9 shows SEM micrographs of OPC1 cells and Ti after 3 days of culture on: (a,b) porous Ti (27\% porosity), showing flattened and well-spread morphology; (c) Ti plate, showing more rounded shape. 
Figure 9. SEM micrographs of OPC1 cells after 3 days of culture on: (a,b) porous Ti (27\% porosity), showing flattened and well-spread morphology; (c) Ti plate, showing more rounded shape [47].
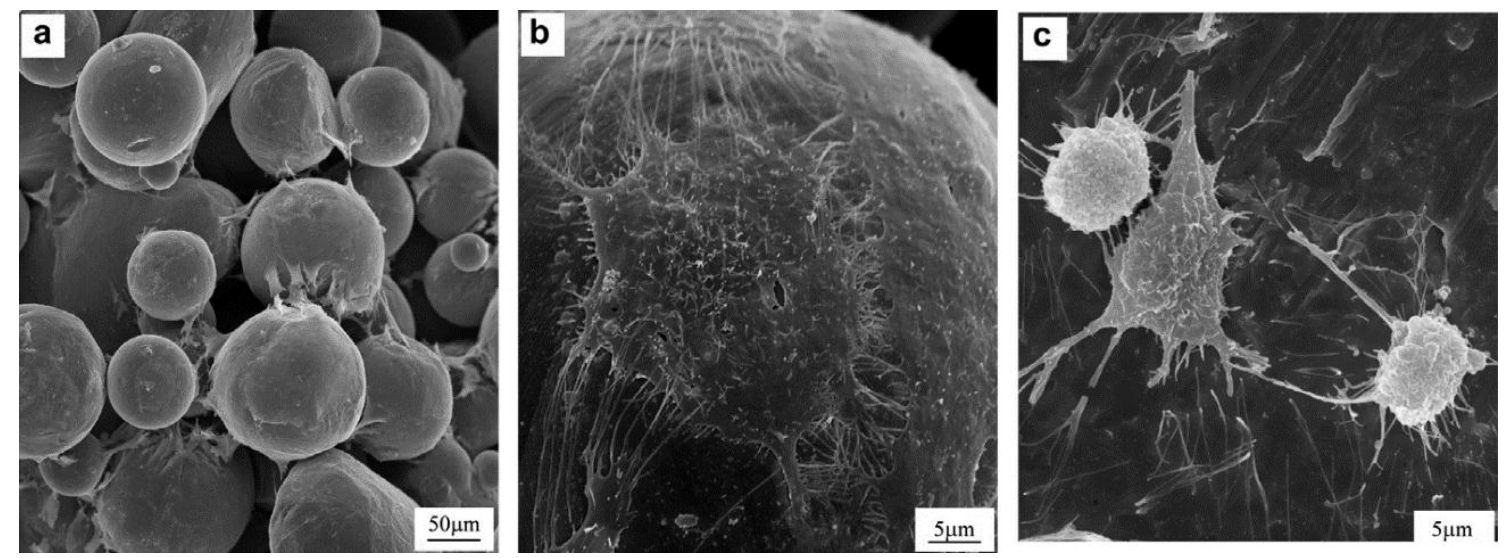

In additive manufacturing, there is another technique which is widely used with polymeric materials. This method is known as fused deposition modelling (FDM). In FDM, the material is extruded in building a part layer by layer from a CAD file. The FDM schematic is shown in Figure 10. This technique also gives room for flexibility in design and this is undoubtedly beneficial for implant fabrication because implant size and shape can be tailored leading to the ability to produce patient specific implants.

Figure 10. Fused deposition modelling schematic.

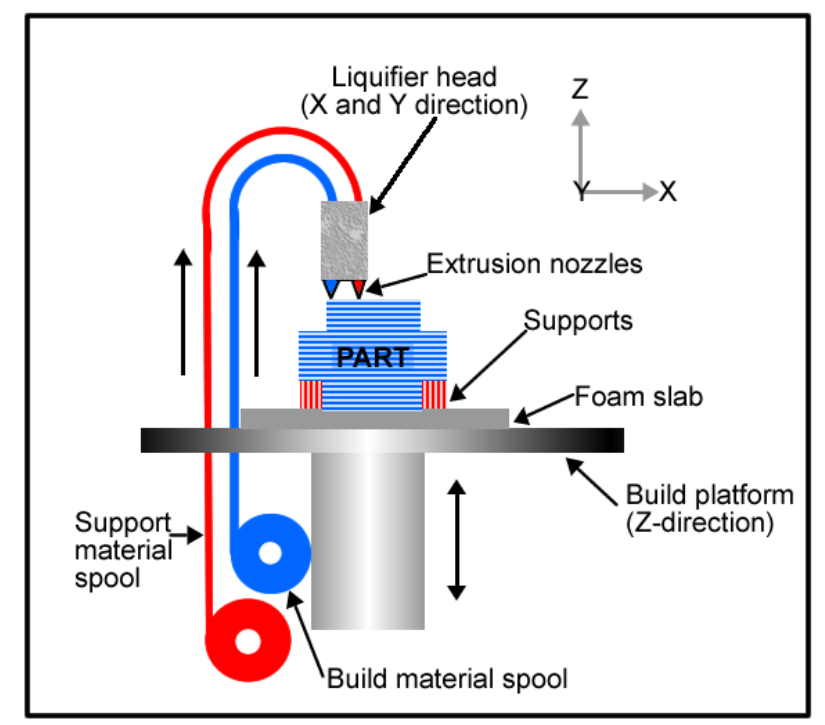

Wiria et al. [49] fabricated titanium implant prototypes using this 3-D printing technique in combination with the debinding and consolidation (sintering) processing that is carried out in MIM. In their study, a 3D Printer normally used for plaster material was used to fabricate green parts of the titanium implant prototype. The CP-Ti powder (325 mesh) was mixed with Poly(vinyl alcohol) (PVA) binder material to make the feedstock. After 3-D printing, the green parts were firstly debound from the PVA binder and then sintered. The subsequent cell culture study which was carried out using the L929 fibroblast cells showed that the porous CP-Ti implant had excellent biocompatibility because of the resulting bone cell attachment and proliferation. The in vitro investigation also revealed increased 
osteogenic differentiation, with little cytotoxicity. Figure 11 shows comparison of cytotoxicity between the CP-Ti scaffolds and control cylinder. The negative control samples used for cytotoxicity tests were in the form of agarose gel cylinders with the same dimension as the titanium implants, whereas phenol samples were used as positive control.

Figure 11. Comparison of cytotoxicity between titanium scaffolds and control cylinder [49].

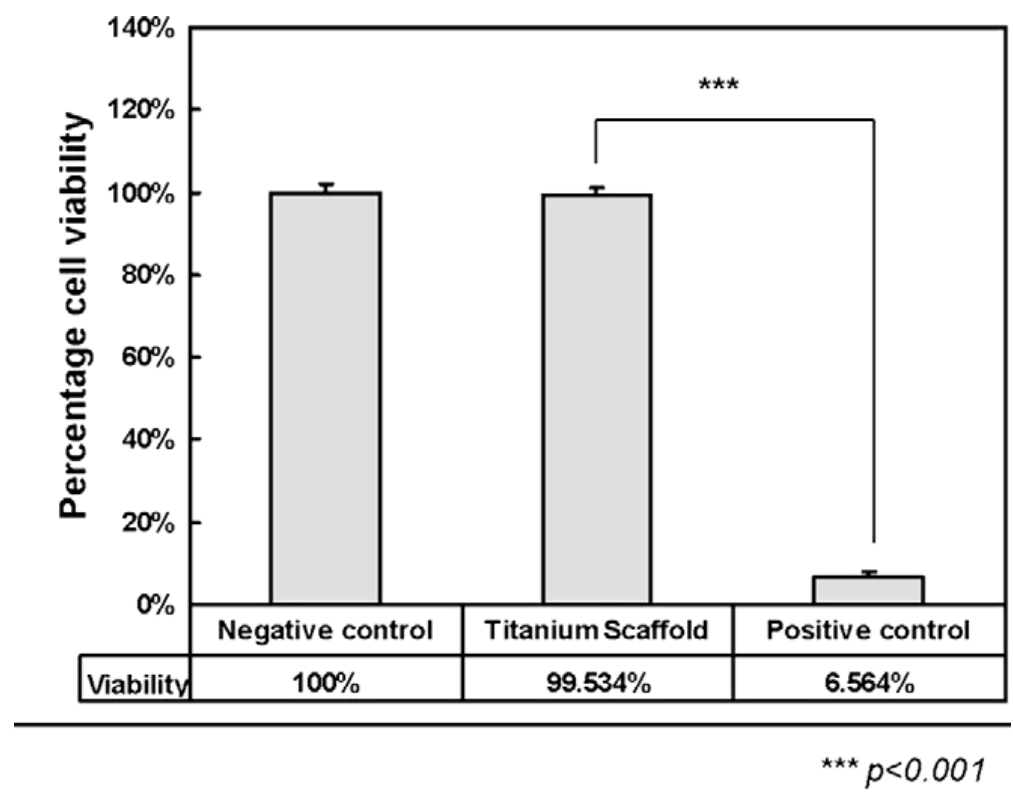

\section{Conclusions and Future Outlook}

Titanium and its alloys have a proven track record as biomedical implants due to their excellent biocompatibility. This is related to the presence of an oxide layer that is typically present on the surface of the material in an oxidising environment. The processing of titanium via the advanced manufacturing technologies of ALM and MIM was until recently inhibited by relatively high costs and the need to demonstrate that the resulting surfaces have the equivalent biocompatibility of implants manufactured using traditional methods. This review has demonstrated that advanced and additive manufacturing can be used successfully to manufacture safe, biocompatible titanium alloy structures for use as medical devices in some applications. This conclusion is supported by a number of in vitro and in vivo studies. The studies used cultured fibroblasts and osteoblasts in the observation of cell responses to surfaces and also human and animal subjects. Several advanced manufacturing routes have also been shown here to provide an enabling environment for the manufacture of implants with tailored surfaces, porosity and scaffolds. For example, this is a feature that has been used in the fabrication of titanium implants where the elastic modulus matches that of bone tissue. Tables 4 and 5 summarise the ALM and MIM processing methods and findings. The processing of titanium alloys manufactured via advanced powder manufacturing routes such as additive layer manufacturing (or 3D printing) and metal injection moulding is clearly receiving increased attention and being adopted as alternative to machining and casting. This is as a result of the associated key advantages which include the design flexibility, reduced processing costs, reduced waste, energy efficiency and improved functionality as has been demonstrated in several of the above studies. Therefore, the advanced manufacturing routes collectively represent a significant opportunity to transform the medical device industry. On the other hand, whilst ALM offers design 
flexibility, the full scale adoption in implant manufacturing may be hindered by the current high cost of machines with metal 3-D printing capabilities and by costs associated with skills training. The field of advanced manufacturing is evolving rapidly, with new technologies and discoveries appearing almost continuously and contributing to a very dynamic field. Furthermore, it has been shown that ALM can produce implants with customised rough surfaces, but in some implant cases such as in joints, a smooth surface is required and ALM and MIM are currently not capable of producing parts with very smooth surface finishes. This means that in some applications, ALM and MIM Ti implants may have to be post-processed or coated to achieve the adequately smooth surfaces. There are also further complexities in that the cell attachment or bone ingrowth, for example, may not be uniform throughout a single $\mathrm{Ti}$ implant part.

Table 4. Summary of ALM processing methods and findings.

\begin{tabular}{|c|c|c|c|c|}
\hline Processing & Alloy & $\begin{array}{c}\text { Biocompatibility } \\
\text { test } \\
\end{array}$ & Cell line/implantation & Other comments and references \\
\hline EBM & Ti6Al4V & In vitro & human fetal osteoblasts (hFOB 1.19) & $\begin{array}{l}\text { Reduced cell proliferation in highly } \\
\text { rough surfaces [33] }\end{array}$ \\
\hline EBM & Ti6Al4V & In vivo & Frontal skull of domestic pig & $\begin{array}{l}\text { More bone contact in more porous } \\
\text { samples [17] }\end{array}$ \\
\hline EBM & Ti6Al4V & In vivo & Rabbit femur and tibia & $\begin{array}{l}\text { As-EBM implant response } \\
\text { comparable to machined [34] }\end{array}$ \\
\hline EBM & Ti6Al4V & In vitro & $\begin{array}{l}\text { Human adipose-derived adult } \\
\text { stem cells (hASC) }\end{array}$ & $\begin{array}{l}\text { Increased proliferation on porous } \\
\text { compared to polished and } \\
\text { unpolished EBM discs [29] }\end{array}$ \\
\hline EBM & Ti6Al4V & In vitro and in vivo & $\begin{array}{l}\text { Osteoblasts extracted from Calvaria } \\
\text { of rabbits, Calvaria of rabbits }\end{array}$ & $\begin{array}{l}\text { Proliferation in porous EBM Ti-64 } \\
\text { implants matched coated implants [35] }\end{array}$ \\
\hline EBM & Ti6Al4V & In vivo & Sheep & $\begin{array}{l}\text { High bone-implant contact in } \\
\text { porous implant [36] }\end{array}$ \\
\hline DMLS & Ti6Al4V & In vitro & human osteoblasts cells (HOB) & $\begin{array}{l}\text { Cultured cells attached and } \\
\text { proliferated on SLM substrates [37] }\end{array}$ \\
\hline DMLS & Unspecified $\mathrm{Ti}$ & In vitro and in vivo & $\begin{array}{l}\text { BMP-7 transduced human } \\
\text { gingival Fibroblasts }\end{array}$ & $\begin{array}{l}\text { In vitro and in vivo test data showing } \\
\text { substantial bone ingrowth [38] }\end{array}$ \\
\hline DMLS & Ti6Al4V & In vivo & $\begin{array}{l}\text { Human anterior mandible, } \\
\text { minipig mandibular }\end{array}$ & $\begin{array}{l}\text { Peri-implant bone in close contact } \\
\text { with the surface of the implant [39] }\end{array}$ \\
\hline DMLS & Ti6Al4V & In vitro & Human osteoblasts & $\begin{array}{l}\text { Osteoblasts well-spread and with } \\
\text { multiple contact points [40] }\end{array}$ \\
\hline LENS & $\mathrm{CP}-\mathrm{Ti}$ & In vitro & human osteoblast cells (OPC1) & Cells well spread on porous Ti [47] \\
\hline LENS & Ti6Al4V & In vivo & Male Sprague-Dawley rats & $\begin{array}{l}\text { Increase in calcium (bone) within } \\
\text { implant pores [48] }\end{array}$ \\
\hline $\begin{array}{l}\text { Modified } \\
\text { FDM }\end{array}$ & $\mathrm{CP}-\mathrm{Ti}$ & In vitro & L929 mouse fibroblast & $\begin{array}{l}\text { Excellent bone cell attachment and } \\
\text { proliferation [49] }\end{array}$ \\
\hline
\end{tabular}


Table 5. Summary of MIM processing and findings.

\begin{tabular}{|c|c|c|c|c|}
\hline Processing & Alloy & Biocompatibility test & Cell line/implantation & Other comments and references \\
\hline MIM & Ti6Al4V & In vitro & L929 (ISO 10993) & Passing results for ISO10993 tests [42] \\
\hline MIM & Ti6Al4V0.5B & In vitro & MG63 cell & $\begin{array}{l}\text { Alloy satisfied requirements of a } \\
\text { MIM implant [28] }\end{array}$ \\
\hline MIM & $\begin{array}{l}\text { CP-Ti and } \\
\text { Ti6Al4V }\end{array}$ & In vitro & MC-3T3-E1 pre-osteoblasts & $\begin{array}{l}\text { Cell adhesion much improved on } \\
\text { the MIM-Ti, BIODIZE }{ }^{\circledR} \text { and } \\
\text { BIOCOAT }^{\circledR}[44]\end{array}$ \\
\hline MIM & CP-Ti & In vitro & MC-3T3-E1 pre-osteoblasts & $\begin{array}{l}\text { MIM-Ti and BIOCER }{ }^{\circledR} \text { had } \\
\text { enhanced cell proliferation, } \\
\text { adhesion and differentiation [45] }\end{array}$ \\
\hline MIM & Ti6Al4V & In vitro and in vivo & $\begin{array}{l}\text { L929 fibroblast and mandible of } \\
\text { Macaca fascicularis }\end{array}$ & $\begin{array}{l}\text { Cells proliferated with filopodia and } \\
\text { attached to MIM Ti-64 [46] }\end{array}$ \\
\hline
\end{tabular}

Published literature clearly demonstrates that advanced powder manufacturing routes produce implants that are suitable for biomedical applications and the field of investigation of the biocompatibility and clinical performance of ALM and MIM metallic implants has been shown in this review to be expanding. However, relative to the biomedical industry and studies, the studies of in vitro biocompatibility, in vivo tissue response, and clinical performance of advanced manufactured surfaces can be considered to be limited in number. There is also room to improve the scope of these studies because of the continuing innovation in advanced manufacturing technologies. To summarise, the available data is encouraging, even where it is not always based on systematic laboratory or detailed clinical studies. There is little doubt that more research is needed into the biocompatibility and functionality of medical devices manufactured using the full range of additive and advanced manufacturing technologies available today.

\section{Acknowledgments}

The author would like to thank the Wellcome Trust for providing financial support through the Institutional Strategic Support Fund (ISSF) to the University of Sheffield to conduct the present research.

\section{Conflicts of Interest}

The author declares no conflict of interest.

\section{References}

1. Elias, C.N.; Lima, J.H.C.; Valiev, R.; Meyers, M.A. Biomedical applications of titanium and its alloys. JOM 2008, 60, 46-49.

2. BomBač, D.; Brojan, M.; Fajfar, P.; Kosel, F.; Turk, R. Review of materials in medical applications. RMZ Mater. Geoenviron. 2007, 54, 471-499.

3. Mueller, E.; Kammula, R.; Marlowe, D. Regulation of biomaterials and medical devices. MRS Bull. 1991, 16, 39-41.

4. Froes, F.H. Titanium powder metallurgy: A review-Part 1. Adv. Mater. Process. 2012, 170, 16-22. 
5. Guo, S.; Qu, X.; He, X.; Zhou, T.; Duan, B. Powder injection molding of Ti-6Al-4V alloy. J. Mater. Process. Technol. 2006, 173, 310-314.

6. Sidambe, A.T.; Figueroa, I.A.; Hamilton, H.G.C.; Todd, I. Metal injection moulding of CP-Ti components for biomedical applications. J. Mater. Process. Technol. 2012, 212, 1591-1597.

7. Quinn, R.K.; Armstrong, N.R. Electrochemical and surface analytical characterization of titanium and titanium hydride thin-film electrode oxidation. J. Electrochem. Soc. 1978, 125, 1790-1796.

8. Schiff, N.; Grosgogeat, B.; Lissac, M.; Dalard, F. Influence of fluoride content and $\mathrm{pH}$ on the corrosion resistance of titanium and its alloys. Biomaterials 2002, 23, 1995-2002.

9. Tengvall, P.; Lundström, I. Physico-chemical considerations of titanium as a biomaterial. Clin. Mater. 1992, 9, 115-134.

10. Paschalis, E.I.; Chodosh, J.; Spurr-Michaud, S.; Cruzat, A.; Tauber, A.; Behlau, I.; Gipson, I.; Dohlman, C.H. In vitro and in vivo assessment of titanium surface modification for coloring the backplate of the boston keratoprosthesis. Investig. Ophthalmol. Vis. Sci. 2013, 54, 3863-3873.

11. Adell, R.; Lekholm, U.; Rockler, B.; Brånemark, P.I. A 15-year study of osseointegrated implants in the treatment of the edentulous jaw. Int. J. Oral Maxillof. 1981, 10, 387-416.

12. Standard Specification for Unalloyed Titanium for Surgical Implant Applications (UNS R50250, UNS R50400, UNS R50550, UNS R50700); ASTM F67-13; American Society for Testing Materials: West Conshohocken, PA, USA, 2013.

13. Standard Specification for Wrought Titanium-6 Aluminum-4 Vanadium ELI(Extra Low Interstitial) Alloy for Surgical Implant Applications (UNS R56401); ASTM F136-13; American Society for Testing Materials: West Conshohocken, PA, USA, 2013.

14. Sidambe, A.T.; Choong, W.L.; Hamilton, H.G.C.; Todd, I. Correlation of metal injection moulded Ti6Al4V yield strength with resonance frequency (PCRT) measurements. Mater. Sci. Eng. A Struct. 2013, 568, 220-227.

15. Zhao, X.; Chen, L.; Xin, L.; Huang, W. Study on microstructure and mechanical properties of laser rapid forming Inconel 718. Mater. Sci. Eng. A Struct. 2008, 478, 119-124.

16. Bidaux, J.E.; Closuit, C.; Rodriguez-Arbaizar, M.; Zufferey, D.; Carreno-Morelli, E. Metal injection moulding of low modulus Ti-Nb alloys for biomedical applications. Powder Metall. 2013, 56, 263-266.

17. Ponader, S.; von Wilmowsky, C.; Widenmayer, M.; Lutz, R.; Heinl, P.; Körner, C.; Singer, R.F.; Nkenke, E.; Neukam, F.W.; Schlegel, K.A. In vivo performance of selective electron beam-melted Ti-6Al-4V structures. J. Bio. Mater. Res. A 2009, 92, 56-62.

18. Parthasarathy, J.; Starly, B.; Raman, S.; Christensen, A. Mechanical evaluation of porous titanium (Ti6Al4V) structures with electron beam melting (EBM). J. Mech. Behav. Biomed. Mater. 2010, 3, 249-259.

19. Murr, L.E.; Amato, K.N.; Li, S.J.; Tian, Y.X.; Cheng, X.Y.; Gaytan, S.M.; Martinez, E.; Shindo, P.W.; Medina, F.; Wicker, R.B. Microstructure and mechanical properties of open-cellular biomaterials prototypes for total knee replacement implants fabricated by electron beam melting. J. Mech. Behav. Biomed. Mater. 2011, 4, 1396-1411.

20. Standard Specification for Metal Injection Molded Titanium-6Aluminum-4Vanadium Components for Surgical Implant Applications; ASTM F2885-11; American Society for Testing Materials: West Conshohocken, PA, USA, 2011. 
21. Standard Specification for Additive Manufacturing Titanium-6 Aluminum-4 Vanadium ELI with Powder Bed Fusion; ASTM F2924-14; American Society for Testing Materials: West Conshohocken, PA, USA, 2014.

22. Standard Specification for Additive Manufacturing Titanium-6 Aluminum-4 Vanadium ELI (Extra Low Interstitial) with Powder Bed Fusion; ASTM F3001-14; American Society for Testing Materials: West Conshohocken, PA, USA, 2014.

23. Sintered-Metal Injection-Moulded Materials-Specifications; ISO 22068-14; International Organisation for Standardisation ISO: Geneva, Switzerland, 2014.

24. Sidambe, A.T.; Figueroa, I.A.; Hamilton, H.G.C.; Todd, I. Taguchi optimization of MIM titanium sintering. Int. J. Powder Metall. 2011, 47, 21-28.

25. Froes, F.H. Titanium powder metallurgy: A review-Part 2. Adv. Mater. Process. 2012, 170, $26-29$.

26. Van Noort, R. The future of dental devices is digital. Dent. Mater. 2012, 28, 3-12.

27. Ferri, O.M.; Ebel, T.; Bormann, R. High cycle fatigue behaviour of Ti-6Al-4V fabricated by metal injection moulding technology. Mat. Sci. Eng. A Struct. 2009, 504, 107-113.

28. Ebel, T.; Blawert, C.; Willumeit, R.; Luthringer, B.J.C.; Ferri, O.M.; Feyerabend, F. Ti-6Al-4V-0.5B: A modified alloy for implants produced by injection molding. Adv. Eng. Mater. 2011, 13, B440-B453.

29. Haslauer, C.M.; Springer, J.C.; Harrysson, O.L.A.; Loboa, E.G.; Monteiro-Riviere, N.A.; Marcellin-Little, D.J. In vitro biocompatibility of titanium alloy discs made using direct metal fabrication. Med. Eng. Phys. 2010, 32, 645-652.

30. Chen, H.; Sago, A.; West, S.; Farina, J.; Eckert, J.; Broadley, M. Biocompatibility of metal injection molded versus wrought ASTM F562 (MP35N) and ASTM F1537 (CCM) cobalt alloys. BioMed. Mater. Eng. 2011, 21, 1-7.

31. Ivanova, O.; Williams, C.; Campbell, T. Additive manufacturing (AM) and nanotechnology: Promises and challenges. Rapid Prototyp. J. 2013, 19, 353-364.

32. Al-Bermani, S.S.; Blackmore, M.L.; Zhang, W.; Todd, I. The origin of microstructural diversity, texture, and mechanical properties in electron beam melted Ti-6Al-4V. Metall. Mater. Trans. A 2010, 41A, 3422-3434.

33. Ponader, S.; Vairaktaris, E.; Heinl, P.; Wilmowsky, C.V.; Rottmair, A.; Körner, C.; Singer, R.F.; Holst, S.; Schlegel, K.A.; Neukam, F.W.; et al. Effects of topographical surface modifications of electron beam melted Ti-6Al-4V titanium on human fetal osteoblasts. J. Biomed. Mater. Res. A 2008, 84A, 1111-1119.

34. Thomsen, P.; Malmström, J.; Emanuelsson, L.; René, M.; Snis, A. Electron beam-melted, free-form-fabricated titanium alloy implants: Material surface characterization and early bone response in rabbits. J. Biomed. Mater. Res. B 2009, 90B, 35-44.

35. Li, X.; Feng, Y.F.; Wang, C.T.; Li, G.C.; Lei, W.; Zhang, Z.Y.; Wang, L. Evaluation of biological properties of electron beam melted Ti6Al4V implant with biomimetic coating in vitro and in vivo. PLos One 2012, 7, e52049, doi:10.1371/journal.pone.0052049.

36. Palmquist, A.; Snis, A.; Emanuelsson, L.; Browne, M.; Thomsen, P. Long-term biocompatibility and osseointegration of electron beam melted, free-form-fabricated solid and porous titanium alloy: Experimental studies in sheep. J. BioMater. Appl. 2013, 27, 1003-1016. 
37. Hollander, D.A.; Wirtz, T.; Walter, M.V.; Linker, R.; Schultheis, A.; Paar, O. Development of individual three-dimensional bone substitutes using "selective laser melting". Eur. J. Trauma 2003, 4, 228-234.

38. Hollister, S.J.; Lin, C.Y.; Saito, E.; Lin, C.Y.; Schek, R.D.; Taboas, J.M.; Williams, J.M.; Partee, B.; Flanagan, C.L.; Diggs, A.; et al. Engineering craniofacial scaffolds. Orthod. Craniofac. Res. 2005, 8, 162-173.

39. Mangano, C.; Piattelli, A.; d'Avila, S.; Iezzi, G.; Mangano, F.; Onuma, T.; Shibli, J.A. Early human bone response to laser metal sintering surface topography: A histologic report. J. Oral Implantol. 2010, 36, 91-96.

40. Warnke, P.H.; Douglas, T.; Wollny, P.; Sherry, E.; Steiner, M.; Galonska, S.; Becker, S.T.; Springer, I.N.; Wiltfang, J.; Sivananthan, S. Rapid prototyping: Porous titanium alloy scaffolds produced by selective laser melting for bone tissue engineering. Tissue Eng. Part C Methods 2009, 15, 115-124.

41. Todd, I.; Sidambe, A.T. Developments in metal injection moulding (MIM). In Advances in Powder Metallurgy: Properties, Processing and Applications; Isaac Chang, Y.Z., Ed.; Woodhead Publishing Limited: Sawston, UK, 2013; pp. 109-146.

42. Sago, J.A.; Broadley, M.W.; Eckert, J.K.; Chen, H. Manufacturing of implantable biomedical devices by metal injection moulding. Adv. Powder Metall. Part Mater. 2010, 4, 89-99.

43. Sago, J.A.; Broadley, M.W.; Eckert, J.K. Metal injection molding of alloys for implantable medical devices. 2012, 48, 41-49.

44. Auzene, D.; Mallejac, C.; Demangel, C.; Lebel, F.; Duval, J.L.; Vigneron, P.; Puippe, J.C. Influence of surface aspects and properties of MIM titanium alloys for medical applications. PIM Int. 2012, 6, 57-61.

45. Demangel, C.; Auzène, D.; Vayssade, M.; Duval, J.-L.; Vigneron, P.; Nagel, M.-D.; Puippe, J.-C. Cytocompatibility of titanium metal injection molding with various anodic oxidation post-treatments. Mater Sci. Eng. C 2012, 32, 1919-1925.

46. Ibrahim, R.; Azmirruddin, M.; Jabir, M.; Muhamad, N.; Rafiq, M.; Hayaty, N.; Kasim, A.; Muhamad, S.; Hanada, K.; Shimizu, T.; et al. Pre-Clinical Study on the Oral Maxillofacial (OMF) Titanium Alloy Implants Produced By Metal Injection Molding (MIM) Using Palm Oil Based Binder System; Euro PM Congress and Exhibition and EPMA: Gothenburg, Sweden, 2013.

47. Xue, W.; Krishna, B.V.; Bandyopadhyay, A.; Bose, S. Processing and biocompatibility evaluation of laser processed porous titanium. Acta Biomater. 2007, 3, 1007-1018.

48. Bandyopadhyay, A.; Espana, F.; Balla, V.K.; Bose, S.; Ohgami, Y.; Davies, N.M. Influence of porosity on mechanical properties and in vivo response of Ti6Al4V implants. Acta Biomater. 2010, 6, 1640-1648.

49. Wiria, F.E.; Shyan, J.Y.M.; Lim, P.N.; Wen, F.G.C.; Yeo, J.F.; Cao, T. Printing of titanium implant prototype. Mater. Des. 2010, 31, S101-S105.

(C) 2014 by the authors; licensee MDPI, Basel, Switzerland. This article is an open access article distributed under the terms and conditions of the Creative Commons Attribution license (http://creativecommons.org/licenses/by/4.0/). 